\title{
"Possible TRALI" developed during bilateral total knee arthroplasty replacement -A case report-
}

\author{
Youn Jin Kim, and Seok Heo \\ Department of Anesthesiology and Pain Medicine, School of Medicine, Ewha Womans University, Seoul, Korea
}

Transfusion-related acute lung injury (TRALI) is one of the leading causes of transfusion-related morbidity and mortality. However, it is frequently not diagnosed and under-reported, which could result in inappropriate treatment. Diagnostic definition for TRALI consists of hypoxia and bilateral pulmonary edema occurring during or within 6 hours of a transfusion in the absence of cardiac failure or intravascular volume overload. Here, we report a fatal case, which resulted from under-recognition and misdiagnosis of TRALI occurring during transfusion with packed red blood cells during a bilateral total knee replacement. (Korean J Anesthesiol 2012; 62: 73-78)

Key Words: Acute lung injury, Pulmonary edema, TRALI, Transfusion.

Homologous blood transfusions cause transfusion reactions such as allergic reactions and fervescence, infectious transmission, acute or delayed hemolytic reactions, and nonhemolytic immune reactions [1]. Recently, the Food and Drug Administration (FDA) reported that transfusion-related acute lung injury (TRALI) is one of the leading causes of transfusionrelated morbidity and mortality [2]. However, the reported incidence is very rare because of clinical under-recognition and misdiagnosis. TRALI is characterized by acute hypoxemia and noncardiogenic pulmonary edema that occur within 6 hours following a transfusion [3]. Although the condition of $80 \%$ of the patients improves within 96 hours by means of conservative treatment, the current reported mortality rate is $5-10 \%$ [4]. Death can result if early recognition, diagnosis, and appropriate treatment are not done.

Here, we report a fatal case, which resulted from the clinical under-recognition and misdiagnosis of a possible case of TRALI occurring during transfusion with packed red blood cells (PRBC) during a bilateral total knee replacement despite ventilator therapy and conservative treatment along with a relevant review of the literature.

\section{Case Report}

A 73-year-old female patient (Height: $160 \mathrm{~cm}$, weight: $58 \mathrm{~kg}$ ) was diagnosed with degenerative osteoarthritis and admitted for bilateral total knee arthroplasty replacement. The patient was previously diagnosed and medicated for hypertension for

Received: April 14, 2011. Revised: 1st, June 21, 2011; 2nd, July 7, 2011. Accepted: July 7, 2011.

Corresponding author: Youn Jin Kim, M.D., Department of Anesthesiology and Pain Medicine, School of Medicine, Ewha Womans University, 911-1, Mok-dong, Yangcheon-gu, Seoul 158-710, Korea. Tel: 82-2-2650-5285, Fax: 82-2-2655-2924, E-mail: ankyj@ewha.ac.kr

(c) This is an open-access article distributed under the terms of the Creative Commons Attribution Non-Commercial License (http:// creativecommons.org/licenses/by-nc/3.0/), which permits unrestricted non-commercial use, distribution, and reproduction in any medium, provided the original work is properly cited. 
5 years, and diagnosed with suspected angina 5 months prior to the surgery receiving aspirin but was discontinued 7 days prior to the surgery. Blood and biochemistry tests done prior to the surgery were within normal limits. The chest radiography examination showed no special findings except cardiomegaly, bronchiectasis or nonspecific fibrosis in right lower lung zone. Upon electrocardiogram examination and echocardiography, a normal sinus rhythm of 66 beats per minute (bpm) along with a nonspecific $\mathrm{T}$ wave abnormality and about $57 \%$ ejection fraction without abnormalities of regional wall motion, thrombi, and vegetation and normal left atrium and ventricle size, normal valve function were revealed.

To perform the surgery under combined spinal epidural anesthesia (CSE), $8 \mathrm{mg}$ of $0.5 \%$ heavy marcaine (Marcaine, Astrazeneca, Sweden) was injected into the subarachnoid space using a needle through the needle technique, and $3 \mathrm{ml}$ of a test dose using $0.375 \%$ of levobupivacaine containing epinephrine ( 1 : 200,000) was given following insertion of an epidural catheter. Surgery began after sensory blocked level reached the T10 dermatome and maintained with intermittent injections of $0.5 \%$ levobupivacaine. The patient was monitored using routine methods [EKG with ST depression, pulse oximeter $\left(\mathrm{SpO}_{2}\right)$, noninvasive blood pressure, arterial blood pressure, urine output (UO), and BIS]. $5 \mathrm{~L} / \mathrm{min}$ of $100 \%$ oxygen was administered via facemask and monitored $\mathrm{SpO}_{2}$ was 98 to $100 \%$, and intermittent midazolam was given for sedation with a BIS level of 65 to 80.

The patient remained hemodynamically stable [Blood pressure (BP); 130-150/70-85 mmHg, heart rate (HR); 65-85 bpm, respiration rate (RR); $15-18 / \mathrm{min}, \mathrm{SpO}_{2} ; 100 \%$ ] through the intraoperative period immediately before tourniquet release of the second knee arthroplasty, and UO was $900 \mathrm{ml}$ during the 5 hours procedure. The estimated blood loss was $400 \mathrm{ml}$, and intraoperative fluid replacement consisted of 1,600 $\mathrm{ml}$ of crystalloid and $500 \mathrm{ml}$ of hetastarch solution. Immediately after tourniquet release of the second knee arthroplasty, the patient was transfused with PRBC. Twenty-five minutes after tourniquet release and initiation of PRBC transfusion, the patient's blood pressure and $\mathrm{SpO}_{2}$ decreased to $87 / 46 \mathrm{mmHg}$ and $90-95 \%$, respectively, with tachypnea and cyanosis so normal saline and a second packed RBC transfusion were rapidly replaced along with a $5 \mathrm{mg}$ injection of ephedrine. Her arterial blood gas analysis (ABGA) showed a pH of 7.32, a $\mathrm{PaCO}_{2}$ of $43 \mathrm{mmHg}$, a $\mathrm{PaO}_{2}$ of $73 \mathrm{mmHg}$, and oxygen saturation $\left(\mathrm{SaO}_{2}\right)$ at $93 \%$. At the end of the operation (about 30 minutes after transfusion), sudden paroxysmal atrial fibrillation developed with systolic BP dropping below $70 \mathrm{~mm} \mathrm{Hg}$, and a $\mathrm{SpO}_{2}$ of $80 \%$ and her mental state changed to drowsy with intact light, eyerish, and gag reflex upon her neurological examination. Respiration and reviving the patient to a conscious state was encouraged, but consciousness continually diminished. Since the oxygen saturation was reduced to $80 \%$ with rigidity of temporomandible joint, etomidate and midazolam were injected, and positive pressure ventilation was performed using a ventilator after endotracheal intubation. The patient's $\mathrm{SpO}_{2}$ increased to 95$98 \%$, but pulmonary edema was suspected because a rale was heard by auscultation from both lungs in addition to the secretion of a frothy sputum. Suddenly, ventricular tachycardia occurred and the HR dropped to less than $30 \mathrm{bpm}$; therefore, cardiopulmonary resuscitation was done. After epinephrine was injected three times and cardioversions with $200 \mathrm{~J}$ biphasic waveform shocks, the patient's rhythm returned to a normal state, and thus, a joint diagnosis was done by the Cardiology and Neurology Departments. There was no specific abnormal findings neurologically, and her transthoracic echocardiography in the OR did not show any fine emboli or embolic mass or pulmonary hypertension or ventricular dysfunction, but did show a decrease in volume and a hyperdynamic status of the
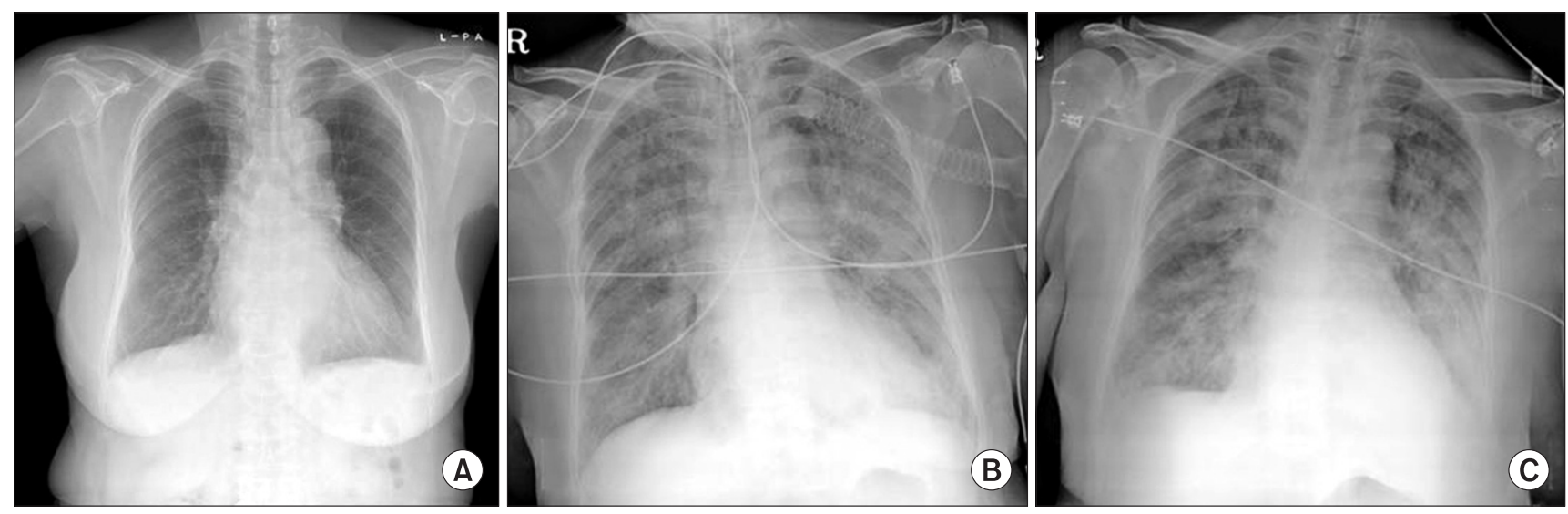

Fig. 1. Chest radiographs. (A) Preoperative, (B) after intubation and CPR, (C) During critical care in ICU. Chest X-ray shows newly appeared bilateral pulmonary infiltrates in (B) and (C) compared with (A). 
heart. Upon portable Chest X-ray examination, it showed bilateral pulmonary infiltrates (Fig. 1). The patient was given 20 mg of furosemide and $500 \mathrm{mg}$ of methylprednisolone IV with inotrophic support (dopamine: $5-15 \mu \mathrm{g} / \mathrm{kg} / \mathrm{min}$, Dobutamine: $5-15 \mu \mathrm{g} / \mathrm{kg} / \mathrm{min}$ ) and manually assisted ventilation for pulmonary edema and $60^{\circ}$ semifowel's position, and yet, her condition of respiratory insufficiency with frothy secretions did not improve over time. She was transferred to ICU for further management by department of cardiogy 1 hour after an advent event. The femoral vein was catheterized, and we continued to focus on treatment for her pulmonary edema, which consisted of medical treatment with furosemide, dopamine: $(10-20 \mu \mathrm{g} /$ $\mathrm{kg} / \mathrm{min})$, dobutamine $(10-20 \mu \mathrm{g} / \mathrm{kg} / \mathrm{min})$, norepinephrine ( $>20 \mu \mathrm{g} / \mathrm{kg} / \mathrm{min}$ ), and volume maintenance using packed RBCs and least crystalloid fluid therapy, but copious pinkish frothy secretions began to flow out from her tracheal tube, and her vital signs and $\mathrm{SpO}_{2}$ were aggravated with anuria and DIC. At this time, her ABGA showed a pH of 7.25, a $\mathrm{PaCO}_{2}$ of $43.8 \mathrm{mmHg}$, a $\mathrm{PaO}_{2}$ of $59.7 \mathrm{mmHg}$, and a $\mathrm{SaO}_{2}$ of $87.7 \%$. Despite her mechanical ventilatory support $\left(\mathrm{F}_{\mathrm{I}} \mathrm{O}_{2}\right.$ of 1.0 with PEEP 10$\left.15 \mathrm{cmH}_{2} \mathrm{O}\right)$ and inotropic treatment, her $\mathrm{SpO}_{2}$ was still low (70-90\%), and her blood pressure $(60-90 / 40-50 \mathrm{mmHg}$ ) was unstable and decreased gradually. ABGA, cardiac enzymes including N-terminal probrain natriuretic peptide BNP (NTproBNP) and Troponin T, and other blood chemistry tests were done (Table 1). EKG and TTE also were done reevaluate and determine the origin of the pulmonary edema. Her cardiac enzymes including Troponin T were slightly increased though her NT-proBNP level was within normal limits, her transthoracic echocardiography in the ICU did not show any signs of heart failure but did show a hyperdynamic and more hypovolemic heart. Although she was again given a total of 7 units of packed RBC for volume maintenance and 2 units of FFP for correction of a coagulation abnormality 3 hours after the initial adverse event during the ICU stay, her HR began to decrease to $<40 \mathrm{bpm}$. She died 1 hour later despite attempts at cardiopulmonary resuscitation.

\section{Discussion}

TRALI has often been discussed in the literature as being related to transfusions. Since there is no consensus with respect to the risk factors and clinical diagnostic criteria of TRALI, recognition of it is confused and not easy in most cases, and thus, only a small percentage of TRALI cases are published. Hence, the incidence of TRALI is estimated to be $0.08-8 \%$ among patients who undergo transfusions $[5,6]$.

The American European Consensus Conference (AECC) modified the definition of TRALI and published it [7], and recently, Canadian Consensus conference further modified the working group criteria for TRALI [8]. The TRALI was classified into "suspected TRALI" and "possible TRALI" and stated that it should be differentially diagnosed from transfusion-associated circulatory overload (TACO). "Suspected TRALI" is defined

Table 1. Laboratory Finding before and after Transfusion

\begin{tabular}{|c|c|c|c|c|c|c|c|}
\hline & \multirow[b]{2}{*}{ Preop } & \multicolumn{3}{|c|}{ Introperative in OR } & \multicolumn{3}{|c|}{ Postoperative in ICU } \\
\hline & & $\begin{array}{c}\text { Before } \\
\text { Tfx. }\end{array}$ & $\begin{array}{c}25 \mathrm{~min} \\
\text { after Tfx. }\end{array}$ & $\begin{array}{c}90 \mathrm{~min} \\
\text { after event }\end{array}$ & $\begin{array}{c}180 \mathrm{~min} \\
\text { after event }\end{array}$ & $\begin{array}{l}210 \mathrm{~min} \\
\text { after event }\end{array}$ & $\begin{array}{c}240 \mathrm{~min} \\
\text { after event }\end{array}$ \\
\hline $\mathrm{pH}$ & 7.47 & 7.40 & 7.32 & 7.25 & 7.25 & 7.25 & 6.939 \\
\hline $\mathrm{PaCO}_{2}(\mathrm{mmHg})$ & 36.5 & 43 & 43 & 35.8 & 43.8 & 42.7 & 75.9 \\
\hline $\mathrm{PaO}_{2}(\mathrm{mmHg})$ & 93.5 & 154 & 73 & 79.4 & 59.7 & 59.6 & 52.2 \\
\hline Base excess & 3.0 & -1.0 & -3.9 & -10.8 & -8.2 & -8.5 & -18.1 \\
\hline $\mathrm{HCO}_{3}{ }^{-}(\mathrm{mmol} / \mathrm{L})$ & 26.2 & 24.3 & 22.2 & 15.2 & 18.6 & 18.1 & 15.4 \\
\hline $\mathrm{SaO}_{2}(\%)$ & 97.5 & 99 & 93 & 94 & 87.7 & 87.6 & 67.3 \\
\hline $\mathrm{Hb}(\mathrm{g} / \mathrm{dl})$ & 13.1 & 10.2 & 10.2 & 11.9 & 11.2 & 16.2 & \\
\hline Platelet & $213 \times 10^{3}$ & & & & $106 \times 10^{3}$ & $79 \times 10^{3}$ & \\
\hline Troponin T (ng/ml) & $<0.010(\mathrm{WNL})$ & & & $0.023(\uparrow)$ & & & \\
\hline $\mathrm{Na} / \mathrm{K}(\mathrm{mEq} / \mathrm{L})$ & $142 / 4.0$ & $136 / 3.4$ & $142 / 3.6$ & $142 / 3.6$ & $148 / 2.5$ & & \\
\hline NT-proBNP & & & & 217 (WNL) & & & \\
\hline PT/aPTT & $105 . / 25.2$ & & & & $16.4 / 43.7$ & $18.0 / 49.2$ & \\
\hline Fibrinogen (mg/dl) & & & & & & $35(\downarrow)$ & \\
\hline $\mathrm{FDP}(\mu \mathrm{g} / \mathrm{ml})$ & & & & & & $127.9(\uparrow)$ & \\
\hline $\mathrm{D}$-dimer $(\mu \mathrm{g} / \mathrm{L})$ & & & & & & $18,300(\uparrow)$ & \\
\hline Transfusion (Unit) & & & PRBC 2 & PRBC 2 & PRBC 3 & $\begin{array}{c}\text { PRBC } 2 \\
\text { FFP } 4\end{array}$ & \\
\hline Treatment & & & Intubation $^{\dagger}$ & $*, \dagger$ † & $\dagger, キ$ & $t, \neq$ & $\dagger, \neq$ \\
\hline
\end{tabular}

Hg: hemoglobin, NT-proBNP: N-terminal probrain natriuretic peptide, PT: prothrombin time, aPTT: activated partial thromboplastin time, FDP: fibrin degradation product, WNL: within normal limits, PRBC: packed red blood cells, FFP: fresh frozen plasma. *Cardioversion: biphasic $200 \mathrm{~J} \rightarrow \mathrm{ROSC}$ at $3 \mathrm{~min}$ after $\mathrm{CPR},{ }^{\dagger}$ semifowerl's position with mechanical ventilator, $\mathrm{FiO}_{2}$ of $1.0, \mathrm{PEEP}$ of $10-15 \mathrm{cmH}_{2} \mathrm{O}$, ${ }^{\dagger}$ used inotrophics with dopamine, dobutamine, norepinephrine and epinephrine. 
as a case in which new acute pulmonary damage occurs as bilateral diffuse infiltration on a chest radiograph during or within six hours after transfusion with a $\mathrm{PaO}_{2} / \mathrm{FiO}_{2}$ ratio less than $300 \mathrm{mmHg}$, hypoxemia of $\mathrm{SpO}_{2}$ less than $90 \%$ in room air, or clinical evidence of hypoxemia in a patient who has not showed any evidence of hypertension related to pulmonary edema or to the left atrium. A case with clinical circulatory overload (e.g. hypertension related to the left atrium) is differentially diagnosed as "TACO". "Possible TRALI" is defined by the same diagnostic criteria with those of the suspected TRALI, but it refers to the case in which the patient has other acute pulmonary risk factors such as sepsis, aspiration, neardrowning, disseminated intravascular coagulation, trauma, pneumonia, drug overdose, fracture, burns, and cardiopulmonary bypass in addition to a transfusion.

It is reported that TRALI occurs within six hours after transfusion, but nonspecific symptoms and signs such as tachypnea, frothy pulmonary secretions, hypotension, hyperthermia, tachycardia, and cyanosis are found within 30-120 minutes after transfusion in most of the cases, and a diffuse rale is heard from both sides by auscultation $[7,8]$. One important factor in TRALI diagnosis is that there should be no evidence of circulatory overload such as S3 gallop or jugular distension. It is known that most of the TRALI patients have euvolemic state or may be hypovolemia because of lung parenchymal excessive fluid leakage. Since the use of diuretics and rapid volume restriction can be the treatment of choice in the case of TACO, they can exacerbate hypovolemia. Accurate diagnosis is important for proper treatment. TRALI's CVP, pulmonary capillary wedge pressure, and B-natriuretic peptide (BNP) values are within the normal ranges. However, TACO and TRALI can be differentiated because TACO shows a BNP measurement of $100 \mathrm{pg} / \mathrm{ml}$ or higher [6,7]. The patient in our case had a NTproBNP value of 227 after the occurrence of TRALI, which was in the normal range, but hypovolemia was exacerbated by the use of diuretics and fluid volume restriction because of the misdiagnosis of not TRALI but TACO by physicians in charge. Consequentially, her vital sign showed still unstable despite the treatment with inotropics and vasoconstrictors, and copious pinkish frothy secretions were persisted. Her prognosis became worse as the unnecessary transfusion was in addition done to correct for unexplained hypovolemia that was later found in the echocardiogram without ever knowing causes.

It is generally known that TRALI occurs usually when FFP is administered, but it can be caused by all the other blood components [4]. The accurate pathogenesis of TRALI is not known, but it has been reported that TRALI is related with antiHLA I, II, anti-neutrophil antibody, and biologically active lipids of the donor plasma [2,6]. Although it is known that TRALI pathogenesis is triggered by a trace amount of plasma, the relationship between the administered dose and the severity is not certain. Rather, hypoxemia is related to the severity. Almost nothing is known about the effects of antibody titer, avidity, or leukocyte-antigen density on the severity or frequency of TRALI reactions, but antibodies were not detected in $17 \%$ of the cases [9]. Hence, considering the amount of time it takes and the cost to obtain results, serological tests are not necessary to diagnose a patient; instead, clinical symptoms are used as the diagnostic criteria.

The FDA recently recommended that clinicians should be alert for TRALI as it publishes its characteristics and morbidity [2]. Kopko and Holland [4] insisted that "possible TRALI" should be kept in mind primarily when respiratory symptoms are accompanied by fever or hypotension within two hours following transfusion. Gajic et al. [6] suggested an approach for the management of respiratory patients after blood transfusion based on the AECC definition [7]. The approach may be helpful to differentiate the diagnosis of TRALI and hydrostatic and permeability pulmonary edema, although both can happen simultaneously in some cases.

Generally, for the treatment of TRALI, transfusions should be stopped; supplemental oxygen should be administered, and auxiliary treatment should be done. It is reported that mechanical support is required in most cases. Different from acute pulmonary damage due to other causes, recovery is rapid with recovery from diffuse pulmonary infiltration known to be within $96 \mathrm{~h}$ following transfusion in $80 \%$ of the cases [4]. The efficacy of steroids is still controversial .

The reason why our case was diagnosed as "possible TRALI" was that the possibility of ALI by fat embolism syndrome (FES) could hardly be excluded. It is known that FES occurs in relationship to many traumatic or nontraumatic conditions, but it usually occurs within 24 to 48 hours after lower extremities long bone fracture operations because of the high intramedullary pressure occurring during prosthesis implantation [10]. Particularly, FES in total knee replacement arthroplasty (TKRA) usually takes place in cemented TKRA that employs a long intramedullary stem, and there is a high probability of pulmonary fat microembolism, especially in the case of bilateral TKRA, in which a tourniquet is installed for a long period $[10,11]$. Since the laboratory and radiographic findings are nonspecific, Gurd's diagnosis criteria based on the clinical symptoms are often used. With these criteria [12], FES can be diagnosed if the symptoms correspond to one major criterion and four minor criteria. In our case, we observed two major criteria including symptoms in the respiratory system and central nervous system, and other minor criteria such as tachycardia, oliguria, thrombocytopenia, and anemia. Various changes in brain functions are found in $86 \%$ of the patients from drowsy to coma. Additionally, it is known that the cutaneous 
symptom, petechial rash, is found at the conjunctiva, around the neck, in the peri-axillary area or upper body within 2436 hours in $20-50 \%$ of the patients, but it spontaneously disappears within one week $[10,11]$. Additional tests were not done to confirm the diagnosis in our case because the patient rapidly exacerbated after the symptoms and eventually died within 7 hours.

In addition, for the diagnosis of TRALI, we should distinguish the changes in hemodynamic status that can develop after deflation of a tourniquet or changes that can develop from movement of thromboemboli or fat particles from the lower part of the body to the right atrium, right ventricle, and pulmonary artery [13]. Especially in surgery performed on the lower part of the body under combined epidural spinal anesthesiasedation with preservation of spontaneous respiration as in our case, respiration and metabolic variables from the release of the tourniquet were promptly recovered within 3-5 minutes from the compensation effect of spontaneous breath [14]. However, the recovery of hypotension was slow due to the loss of sympathetic tone. The decrease in blood pressure that develops after the deflation of the tourniquet was significantly low 3 minutes after release due to a loss of blood, reflexive vascular relaxation of the ischemic area, and systemic inflow of metabolites produced from anaerobic metabolism such as adenosine and lactic acid. This can be suppressed up to 15 minutes after release. However, it is known that any tendency of hypotension can be simply managed through vasopressor agents. In our patient, the $\mathrm{pH}, \mathrm{PaCO}_{2}$, potassium, and lactic acid measured after the release of the second TKRA tourniquet were within normal range, which rules out the possibility of hypotension from the expansion of the blood vessels and myocardial depression. In addition, since the last injection of the epidural local anesthetic was 55 minutes before the deflation of the tourniquet, which was 80 minutes before the event occurred, the possibility of sympathetic block influence is thought to be negligible. Park and Kim [15] reported that in cases of bilateral TKRA, the decrease in cardiac index and mean arterial pressure is larger in the second TKRA compared to the first; thus, properly maintaining preload is very important. When injection of crystalloid is increased to the speed of $20 \mathrm{ml} /$ $\mathrm{kg} / \mathrm{h}$ after deflation of the tourniquet and 3 units of PRBC is transfused to increase the full load, there were no differences in arterial pressure after the deflation of the tourniquet. In our case, hypotension and arrhythmia occurred 25 minutes after the deflation of the tourniquet; therefore, the volume state of the patient was euvolemia, and according to the duration of time, we suspected that recovery from tourniquet syndrome was possible. In addition, it is necessary to distinguish whether it was hydrostatic pulmonary edema from circulatory overload or permeability pulmonary edema from ALI to determine the cause of hypoxia and pulmonary edema that developed in our patient; thus, the possibility of circulatory and respiratory suppression from the tourniquet seems unlikely.

The patient in our case had been taking aspirin since angina was suspected by a local clinic because of dyspnea that had occurred five months before. The joint diagnosis with the Department of Cardiology did not show any distinctive cardiovascular symptoms, and the electrocardiography and echocardiography did not show any specific findings. The cardiac enzyme levels were within the normal limits indicating that there would be no specific problems during the operation, and the chest pulmonary radiograph did not show any findings by which acute pulmonary damage could be suspected. However, before the end of the operation, just over five hours, that is, 25 minutes after the transfusion, symptoms began to appear such as hypotension, tachycardia, and cyanosis immediately followed by diminished consciousness, tachypnea, frothy pulmonary secretions, and arrhythmia. The possibility of "Possible TRALI" was un-recognized and misdiagnosed. Since the auxiliary treatment was originally for cardiogenic pulmonary edema, we did mechanical ventilator therapy, loop diuretics, inotropics, and crystalloids volume restriction. Additionally, two or three units of blood were transfused to maintain the circulatory volume, and even two units of FFP were administered to correct the DIC one hour before respiratory arrest occurred. These treatments might have exacerbated the symptoms rather than providing conservative treatments for the patient.

Conclusively, clinicians should make decisions about transfusions for their patients using an appropriate criterion. TRALI has been the major cause of transfusion-related morbidity and mortality, recently. Differential diagnosis of TRALI from TACO and other diseases is difficult since it is suspected and diagnosed by the clinical symptoms and findings; use of consensus definitions that were recently introduced can help to improve the prognosis of TRALI patients by doing the diagnosis and treatment early. Moreover, blood should be transfused to patients based on strict strategies so that transfusion-related complications including TRALI can be reduced.

\section{References}

1. Drummond JC, Petrovitch CT, Lane TA. Hemostasis and transfusion medicine. In: Clinical Anesthesia. 6th ed. Edited by Barash PG, Cullen BF, Stoelting RK, Cahalan MK, Stock MC: Philadephia, Lippincott-Raven Publishers. 2009, pp 369-78.

2. Goldman M, Webert KE, Arnold DM, Freedman J, Hannon J, Blajchman MA, et al. Proceedings of a consensus conference: towards an understanding of TRALI. Transfus Med Rev 2005; 19: $2-31$. 
3. Toy P, Popovsky MA, Abraham E, Ambruso DR, Holness LG, Kopko PM, et al. Transfusion-related acute lung injury: definition and review. Crit Care Med 2005; 33: 721-6.

4. Kopko PM, Holland PV. Transfusion-related acute lung injury. Br J Haematol 1999; 105: 322-9.

5. Silliman CC, Boshkov LK, Mehdizadehkashi Z, Elzi DJ, Dickey WO, Podlosky L, et al. Transfusion-related acute lung injury: epidemiology and a prospective analysis of etiologic factors. Blood 2003; 101: 454-62.

6. Gajic O, Gropper MA, Hubmayr RD. Pulmonary edema after transfusion: how to differentiate transfusion-associated circulatory overload from transfusion-related acute lung injury. Crit Care Med 2006; 34(5 Suppl): S109-13.

7. Bernard GR, Artigas A, Brigham KL, Carlet J, Falke K, Hudson L, et al. The American-European Consensus Conference on ARDS. Definitions, mechanisms, relevant outcomes, and clinical trial coordination. Am J Respir Crit Care Med 1994; 149: 818-24.

8. Kleinman S, Caulfield T, Chan P, Davenport R, McFarland J, McPhedran S, et al. Toward an understanding of transfusion-related acute lung injury: statement of a consensus panel. Transfusion
2004; 44: 1774-89.

9. Tiyerili V, Becher UM, Strach K, Mueller CF, Nickenig G, Schwab JO. Unclear acute respiratory failure in a 64-year-old woman after coronary intervention. Dtsch Med Wochenschr 2010; 135: 2235-8.

10. Koessler MJ, Pitto RP. Fat and bone marrow embolism in total hip arthroplasty. Acta Orthop Belg 2001; 67: 97-109.

11. Johnson MJ, Lucas GL. Fat embolism syndrome. Orthopedics 1996; 19: 41-8.

12. Gurd AR, Wilson RI. The fat embolism syndrome. J Bone Joint Surg Br 1974; 56B: 408-16.

13. Kam PC, Kavanagh R, Yoong FF. The arterial tourniquet: pathophysiological consequences and anaesthetic implications. Anaesthesia 2001; 56: 534-45.

14. Takahashi S, Mizutani T, Sato S. Changes in oxygen consumption and carbon dioxide elimination after tourniquet release in patients breathing spontaneously under epidural anesthesia. Anesth Analg 1998; 86: 90-4

15. Park SJ, Kim SY. Hemodynamic and metabolic changes induced by tourniquet release during both total knee replacement arthroplasty. Korean J Anesthesiol 2003; 45: 47-53. 